Article

\title{
The Long Non-Coding RNA RHPN1-AS1 Promotes Uveal Melanoma Progression
}

\author{
Linna Lu ${ }^{\dagger}$, Xiaoyu $\mathrm{Yu}^{\dagger}$, Leilei Zhang ${ }^{\dagger}$, Xia Ding, Hui Pan, Xuyang Wen, Shiqiong Xu, Yue Xing, \\ Jiayan Fan, Shengfang Ge, He Zhang, Renbing Jia * and Xianqun Fan *
}

Department of Ophthalmology, Ninth People's Hospital, Shanghai Jiao Tong University School of Medicine, Shanghai 200025, China; drlulinna@126.com (L.L.); yuxiaoyunzb@163.com (X.Y.); a789leilei@126.com (L.Z.); abcdingxia@126.com (X.D.); xypanhui@163.com (H.P.); winston-a@hotmail.com (X.W.); 15721201988@163.com (S.X.); fishashorejessica@163.com (Y.X.); fanjiayan1118@126.com (J.F.); geshengfang@sjtu.edu.cn (S.G.); zhanghe@sjtu.edu.cn (H.Z.)

* Correspondence: jrb19760517@hotmail.com (R.J.); fanxq@sh163.net (X.F.);

Tel./Fax: +86-138-1856-7505 (R.J.); +86-21-6313-5606 (X.F.)

+ These authors contributed equally to this work.

Academic Editor: Martin Pichler

Received: 30 December 2016; Accepted: 16 January 2017; Published: 23 January 2017

\begin{abstract}
Increasing evidence suggests that aberrant long non-coding RNAs (lncRNAs) are significantly correlated with the pathogenesis, development and metastasis of cancers. RHPN1 antisense RNA 1 (RHPN1-AS1) is a 2030-bp transcript originating from human chromosome 8q24. However, the role of RHPN1-AS1 in uveal melanoma (UM) remains to be clarified. In this study, we aimed to elucidate the molecular function of RHPN1-AS1 in UM. The RNA levels of RHPN1-AS1 in UM cell lines were examined using the quantitative real-time polymerase chain reaction (qRT-PCR). Short interfering RNAs (siRNAs) were designed to quench RHPN1-AS1 expression, and UM cells stably expressing short hairpin (sh) RHPN1-AS1 were established. Next, the cell proliferation and migration abilities were determined using a colony formation assay and a transwell migration/invasion assay. A tumor xenograft model in nude mice was established to confirm the function of RHPN1-AS1 in vivo. RHPN1-AS1 was significantly upregulated in a number of UM cell lines compared with the normal human retinal pigment epithelium (RPE) cell line. RHPN1-AS1 knockdown significantly inhibited $\mathrm{UM}$ cell proliferation and migration in vitro and in vivo. Our data suggest that RHPN1-AS1 could be an oncoRNA in UM, which may serve as a candidate prognostic biomarker and target for new therapies in malignant UM.
\end{abstract}

Keywords: lncRNA; RHPN1-AS1; uveal melanoma; migration

\section{Introduction}

Uveal melanoma (UM) is the most common primary intraocular tumor in adults, which arises from uveal melanocytes and has a strong propensity to metastasize [1]. The most frequent metastatic site is the liver, followed by the lung and soft tissues [2]. Although optimal treatments (surgery or radiation) have been developed for primary tumors, there are no effective therapies for metastatic UM. In the Collaborative Ocular Melanoma Study, the prognosis for metastatic UM was found to be poor, with a one-year overall mortality rate of $80 \%-87 \%[3,4]$. Highly metastatic UM tumors are usually caused by the loss of one copy of chromosome 3 and the gain of an additional 8q [5]. Recent studies have shown that somatic mutations occur in a mutually exclusive pattern in Guanine Nucleotide-Binding Protein $\alpha-\mathrm{Q}(G N A Q)$ or Guanine Nucleotide-Binding Protein G $\alpha-11$ (GNA11) in $\sim 83 \%$ of UM cases [6], and inactivating somatic mutations of BRCA associated protein-1 (BAP1) occur in $\sim 84 \%$ of metastasizing tumors [7]. 
Research on mammalian transcriptomes suggests that only $1.5 \%$ of the human genome encodes protein-coding genes [8]. However, recent data from the Encyclopedia of DNA Elements (ENCODE) consortium indicate that around $70 \%$ of human genome is actively transcribed, generating a vast range of non-coding RNAs (ncRNAs) [9]. Based on the transcript length, ncRNAs are classified into small ncRNAs (<200 nt) and long ncRNAs (lncRNAs, $>200 \mathrm{nt})$. Although lncRNAs share common features with mRNAs, as many of them are transcribed by RNA polymerase II, spliced, and 5'-capped [9], lncRNAs also have several distinct features. Some lncRNAs are evolutionarily conserved, implying that they are functionally important [10]. Genome screening studies indicate that lncRNAs are often expressed in a tissue-, developmental stage- or disease-specific pattern [11]. In addition, there is evidence indicating that lncRNAs are important regulatory molecules at various levels, including involvement in chromatin modification, transcription, and post-transcriptional processing [8]. Previous studies have shown that the lncRNA ROR occupies and activates the tescalcin (TESC) promoter and promotes metastasis [12]. However, the function of lncRNAs in UM is not well understood.

Aberrant expression of IncRNAs has been shown to contribute to tumorigenesis in cancers such as prostate cancer, gastric cancer and leukemia [13-15], and we previously conducted a study of cDNA microarrays in UM samples and normal tissues (data unpublished). We found that RHPN1 Antisense RNA 1 (RHPN1-AS1) was highly expressed in UM cancerous tissues compared to normal tissues. Down regulating RHPN1-AS1 in a variety of UM cells inhibitedcolony formation, migration and invasionin vitroand tumor growthin vivo. Our results show, for the first time, that RHPN1-AS1 plays a potential role in the progression of UM. Thus, this lncRNA might be an attractive biomarker and therapeutic target in UM.

\section{Results}

\subsection{RHPN1-AS1 Is a Cytoplasmic IncRNA That Is Upregulated in Uveal Melanoma (UM)}

To investigate the expression profiling and the role of lncRNAs in UM, microarrays containing probes targeting 12,784 lncRNAs were used in uveal melanoma samples and noncancerous tissues (unpublished data). We found that the expression level of RHPN1-AS1 was upregulated in UM tissues compared with normal tissues. Furthermore, the expression level of RHPN1-AS1 was detected by qRT-PCR in a variety of UM cell lines, including OCM1 and OM431. RPE cells served as controls. Compared with the normal RPE cells, RHPN1-AS1 was significantly overexpressed in UM cells (Figure 1A).

The non-coding nature of RHPN1-AS1 was confirmed by coding-potential analysis (Figure S1). Prediction of putative proteins encoded by RHPN1-AS1 using Open Reading Frame Finder and the condon substitution frequency scores (CSF) of RHPN1-AS1 indicated that RHPN1-AS1 lacks protein coding potential. Next, to determine the subcellular localization of RHPN1-AS1, we performed RNA fluorescence in situ hybridization (FISH) analyses using cy3-labeled probes that recognize RHPN1-AS1. We found that fluorescent signals (red) appeared in cytoplasm (Figure 1B), suggesting that RHPN1-AS1 is located in the cytoplasm. This was further confirmed by nuclear/cytoplasmic RNA fractionation, which revealed that RHPN1-AS1 is associated with the cytoplasmic fractions (Figure 1C).

\subsection{RHPN1-AS1 Knockdown Cell Lines}

To decipher the potential role of RHPN1-AS1 in UM, three siRNAs were designed to knockdown RHPN1-AS1 expression. RHPN1-AS1 expression was significantly knocked down after transfection with these siRNAs in OM431 and OCM1 cells. The highest interference rate was about 70\% in OM431 cells and 60\% in OCM1 cells ( $p<0.05$ vs. controls and Mock, Figure 1D,E). RHPN1-AS1-si1 and RHPN1-AS1-si2 were was selected for use in subsequent experiments. 
A

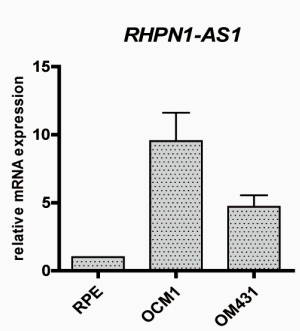

B

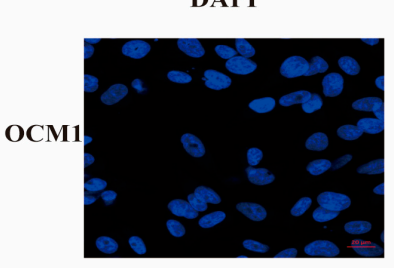

RHPN1-AS1

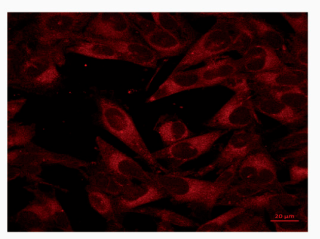

MERGE

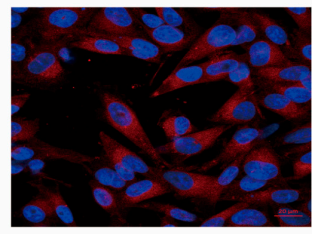

$\boldsymbol{E}$
OM431

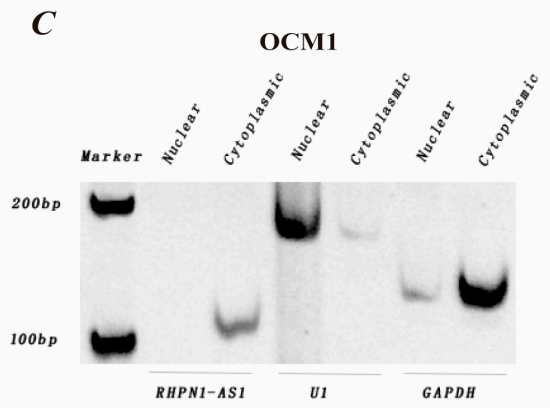

D

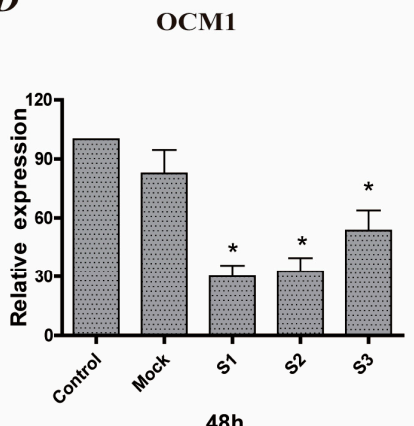

$48 \mathrm{~h}$

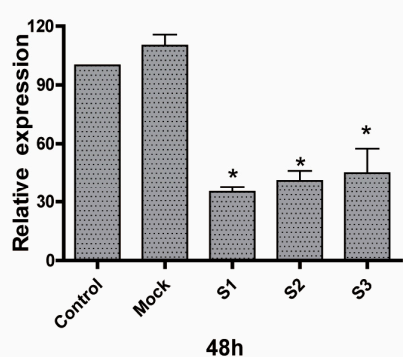

Figure 1. RHPN1-AS1, a cytoplasmic lncRNA, is aberrantly expressed in UM cell lines. (A) RHPN1-AS1 expression was measured by real-time PCR in different UM cells and normal cell (RPE). RHPN1-AS1 presented higher expression in melanoma cell lines OCM1 and OM431 than RPE cells; (B) RHPN1-AS1 is cytoplasmically distributed. RNA fluorescence in situ hybridization (FISH) (red) was performed with cy3-labeled probes that recognizing RHPN1-AS1. The scale bars represent $20 \mu \mathrm{m}$; (C) RHPN1-AS1 is associated with the cytoplasmic fractions. Total RNAs from OCM1 cells were separated into cytoplasmic and nuclear soluble fractions. U1, GAPDH were used as controls; (D,E) The interference rate was detected $48 \mathrm{~h}$ after RHPN1-AS1 siRNAs transfection in OCM1 and OM431 cells. Triplicate assays were performed for each sample and the relative level of RHPN1-AS1 was normalized to the GAPDH. $\left({ }^{*} p<0.05\right)$.

The pGIPZ-shRNA vectors and empty pGIPZ control vectors with an enhanced green fluorescent protein (EGFP) maker was packaged into lenti-viruses and transfected human OCM1 and OM431 cells, named RHPN1-AS1-sh1, RHPN1-AS1-sh2 and mock, respectively. Transfection efficiency was determined by GFP expression. GFP expression was seen in the mock, RHPN1-AS1-sh1 and -sh2 cells (Figure 2A). The knockdown efficiency was measured by qRT-PCR. RHPN1-AS1 expression was significantly decreased in RHPN1-AS1-sh1 and RHPN1-AS1-sh2 transfected cells (Figure 2B).

\subsection{Down-Regulation of RHPN1-AS1 Inhibited Cell Proliferation, Migrationand Invasion In Vitro}

Next, we investigated whether the characteristics of the tumor cells were altered after RHPN1-AS1 knockdown. We first examined the colony formation ability of shRHPN1-AS1-OCM1 cells and shRHPN1-AS1-OM431 cells compared to the control and mock groups using the colony formation assay. The number of colonies was significantly decreased after RHPN1-AS1 knockdown $(p<0.05$, Figure 3A). We also examined the effect of RHPN1-AS1 knockdown on the migration and invasion ability of UM cells. The results demonstrated that the RHPN1-AS1 knockdown inhibited UM cell migration by $\sim 55 \%$ in OCM1 cells and by $\sim 50 \%$ in OM431 cells, respectively ( $p<0.05$, Figure 3B) using trans-well assay. The matrigel invasion assay also showed that RHPN1-AS1 knockdown in UM cells caused a significant decrease in cell invasion $(p<0.05$, Figure 3C). These data indicate that RHPN1-AS1 plays a regulatory role in tumor progression. 
A

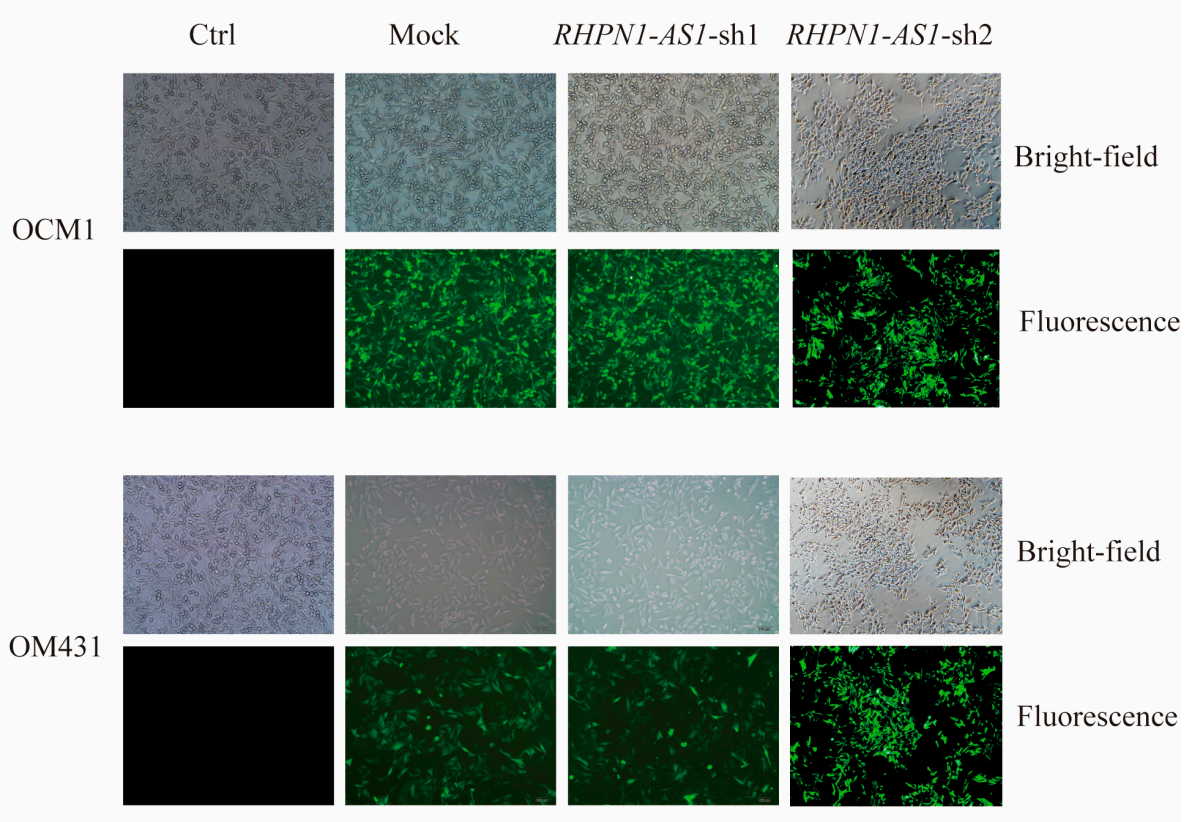

B

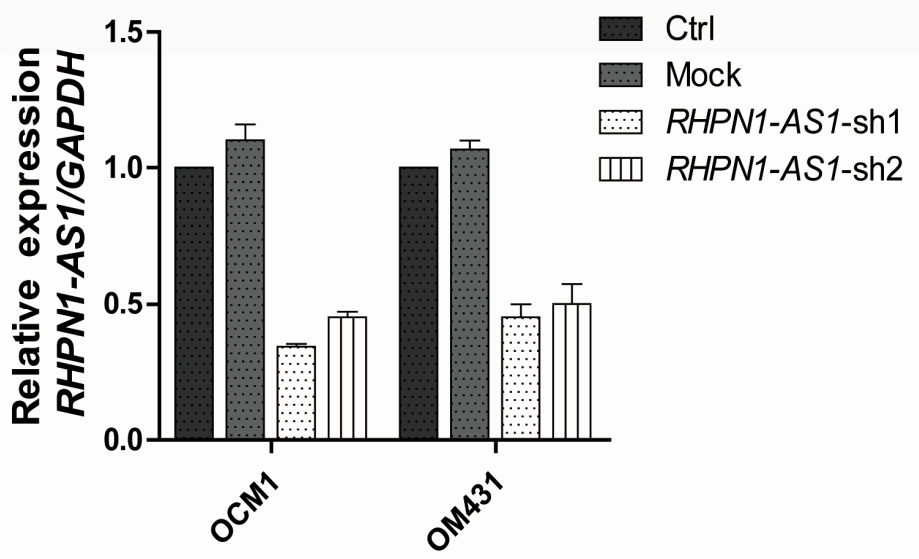

Figure 2. RHPN1-AS1 knockdown by two shRNAs: (A) EGFP was used to track the expression of RHPN1-AS1 shRNAs and control vectors in OCM1 and OM431 cells. The scale bars represent $100 \mu \mathrm{m}$; and (B) detection of RHPN1-AS1 mRNA level in OCM1 and OM431 cells after shRNA-mediated knockdown of RHPN1-AS1 by qRT-PCR.

\subsection{Down-Regulation of RHPN1-AS1 Decreased Xenograft Growth In Vivo}

To examine the biological significance of RHPN1-AS1 on tumor growth in vivo, we established a xenograft model in nude mice; $1 \times 10^{6}$ of the control OCM1 or RHPN1-AS1-sh1OCM1 cells were subcutaneously injected into the right flanks of mice. The tumor volume was measured once every 3-4 days. Fourteen days after injection, the tumor growth of RHPN1-AS1-sh1OCM1 cells was significant slower than that of control cells $(p<0.05$, Figure $4 \mathrm{~A})$. After 28 days, the mice were sacrificed, and the tumors were removed and analyzed (Figure 4B). The average tumor weight of RHPN1-AS1-sh1OCM1 cells was significantly lower than that of the control cells $(p<0.05$, Figure $4 C)$. These data indicate that the growth of the tumors was impaired after RHPN1-AS1 knockdown in vivo. 
A
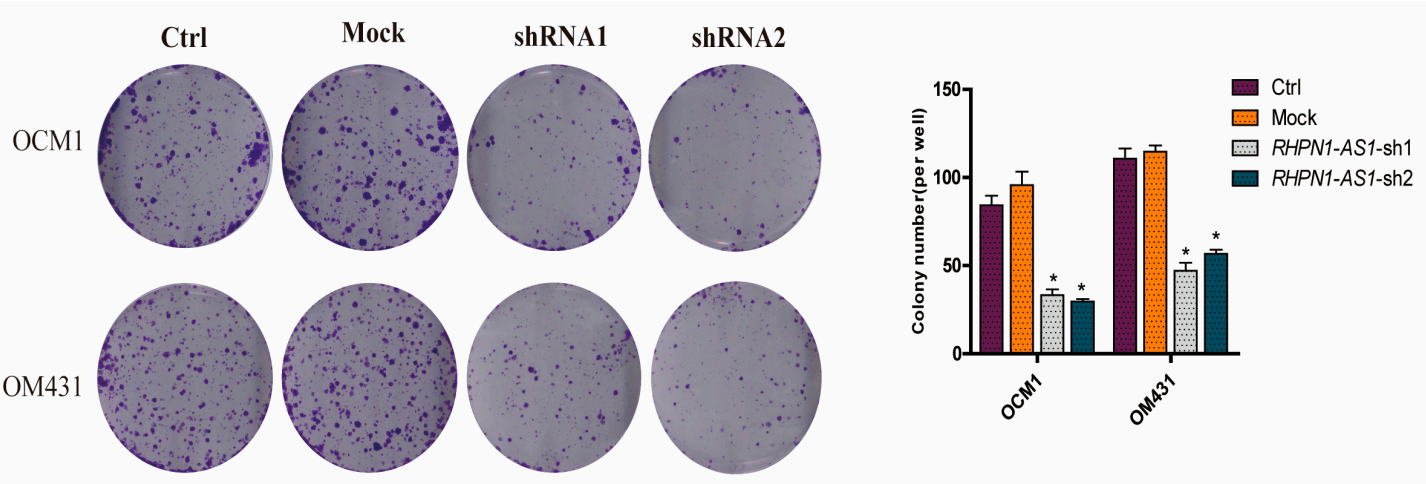

B
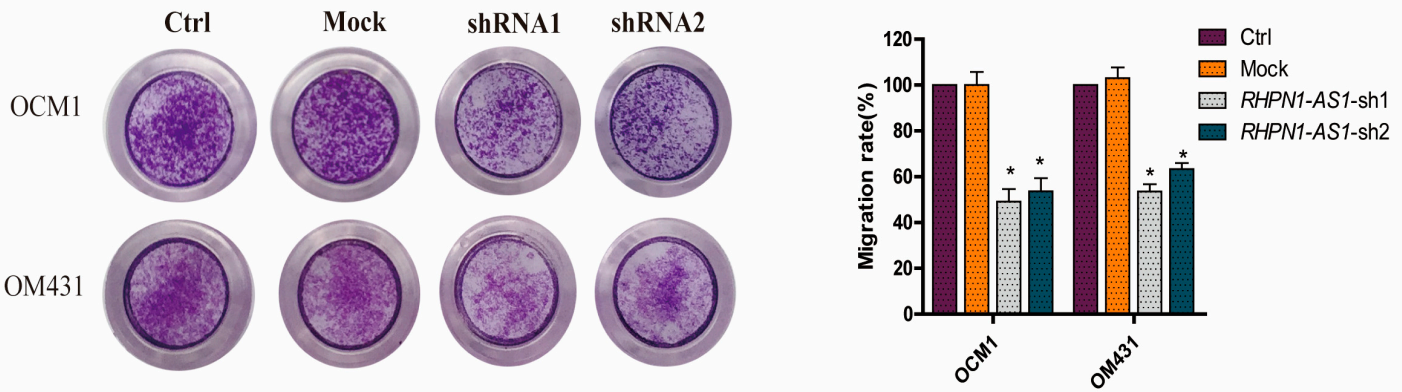

C

$\operatorname{Ctr}$

Mock

shRNA1

ShRNA2
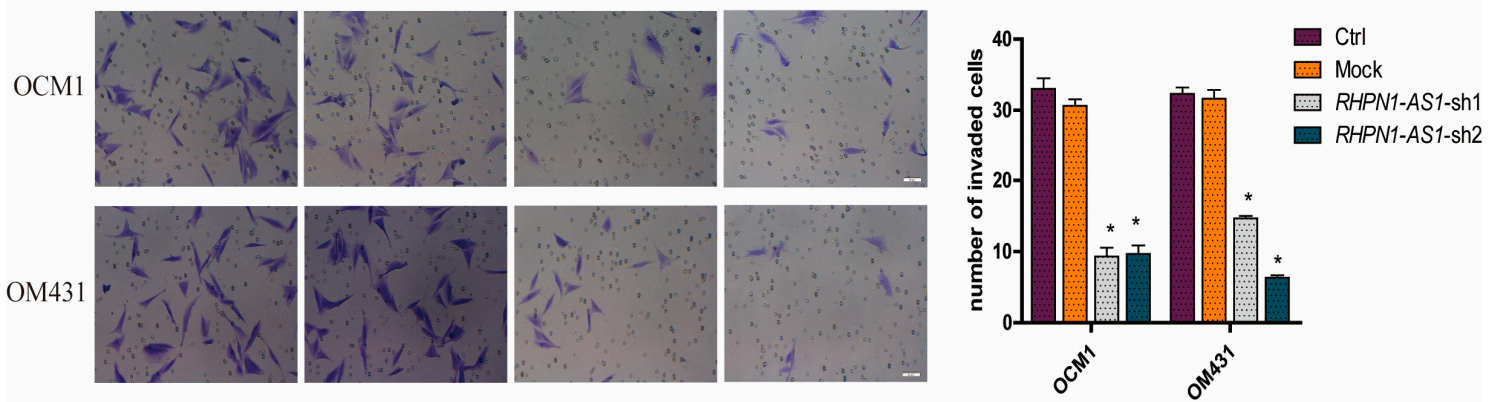

Figure 3. Knockdown of RHPN1-AS1 inhibits proliferation, migration and invasion of UM cells in vitro. (A) Colony formation assays were performed to evaluate the effect of RHPN1-AS1 on growth of UM cells after knockdown RHPN1-AS1. ${ }^{*} p<0.05$; (B) The effect of RHPN1-AS1 downregulation on the migration ability of OCM1 and OM431 cells was assessed using transwell assay. ${ }^{*} p<0.05$; (C) Effects of RHPN1-AS1 knockdown on the invasion potency of OCM1 and OM431 cells were detected using matrigel invasion assay. The scale bars represent $50 \mu \mathrm{m} .{ }^{*} p<0.05$.

\subsection{Gene Expression Profile Analysis Revealed That Angiogenesis and Multiple Pathways May Be Downstream Targets Affected by RHPN1-AS1}

To elucidate the mechanisms by which RHPN1-AS1 contributes to the progression of UM, we carried out microarray analysis comparing the gene expression of OCM1 cells versus OCM1 cells stably transfected with RHPN1-AS1-sh1. Differentially expressed genes with at least a two-fold change were identified. The expression of 136 genes was altered compared with untreated tumor cells (Table S1). We functionally annotated these differentially expressed genes using gene ontology (GO). The categories involved angiogenesis, cell adhesion and extracellular matrix organization (Figure 5A,B). In addition, we identified significant pathways that mediated the functions of the differentially expressed genes based on the Kyoto Encyclopedia of Genes and Genomes (KEGG) database. The most 
significantly changed pathways included bladder cancer, nicotinate and nicotinamide metabolism, and TGF- $\beta$ signaling pathway (Figure 5C). Notably, genes involved in TGF- $\beta$ signaling pathway were significantly enriched, suggesting that RHPN1-AS1 may participate in the TGF- $\beta$ signaling pathway thus contributing to epithelial-to-mesenchymal transition (EMT). However, further studies are still needed to elucidate the mechanisms by which RHPN1-AS1 modulates those processes and pathways.
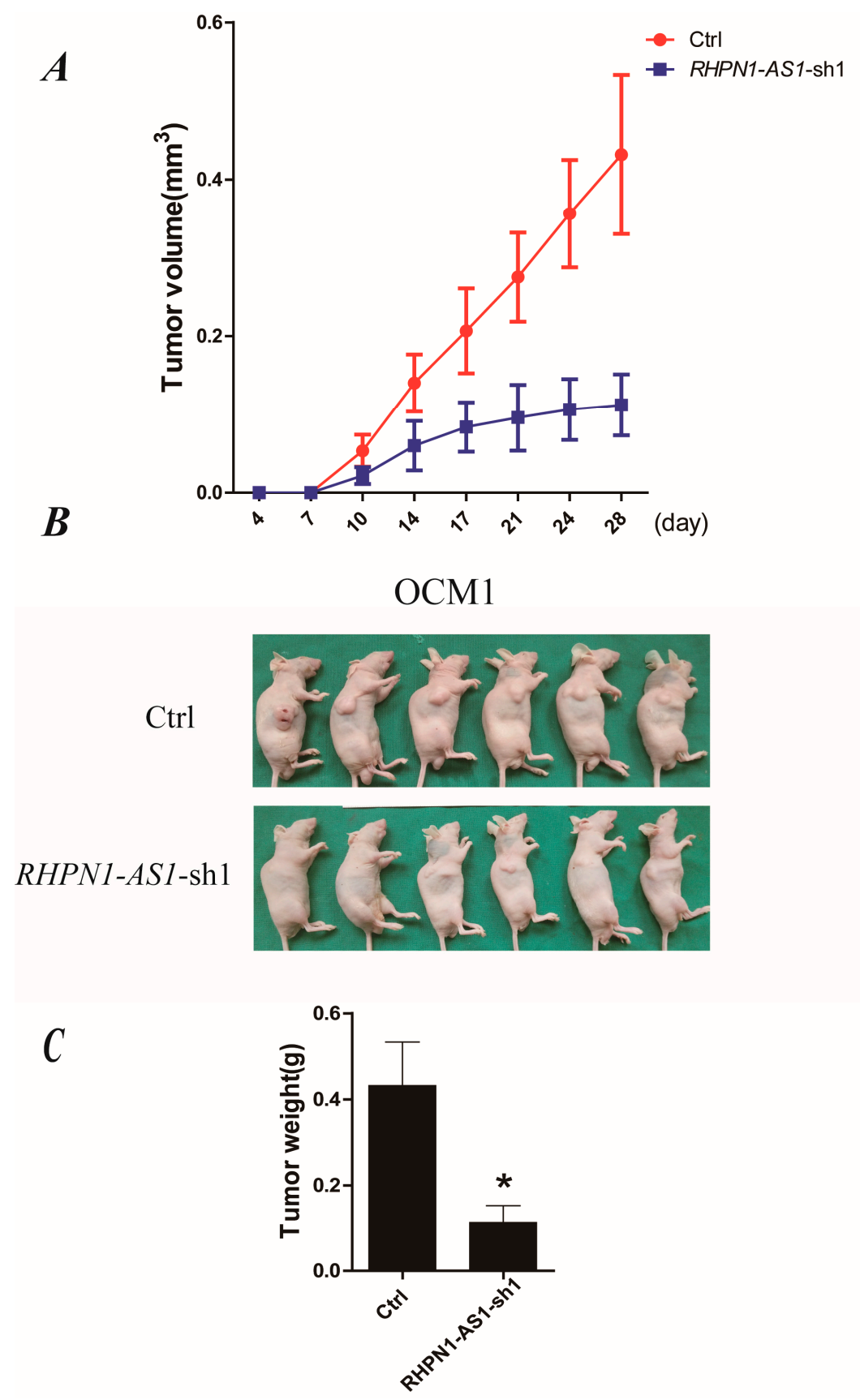

Figure 4. Depressed expression of RHPN1-AS1 repressed UM cell growth in vivo. (A) Tumor volume growth curves. Each data point represents the mean \pm SD; (B) RHPN1-AS1 knockdown OCM1 and control cells were injected into nude mice subcutaneously. Representative images of tumor growth 28 days after subcutaneous injection; (C) Mean tumor weights four weeks after inoculation. * $p<0.05$. 
A

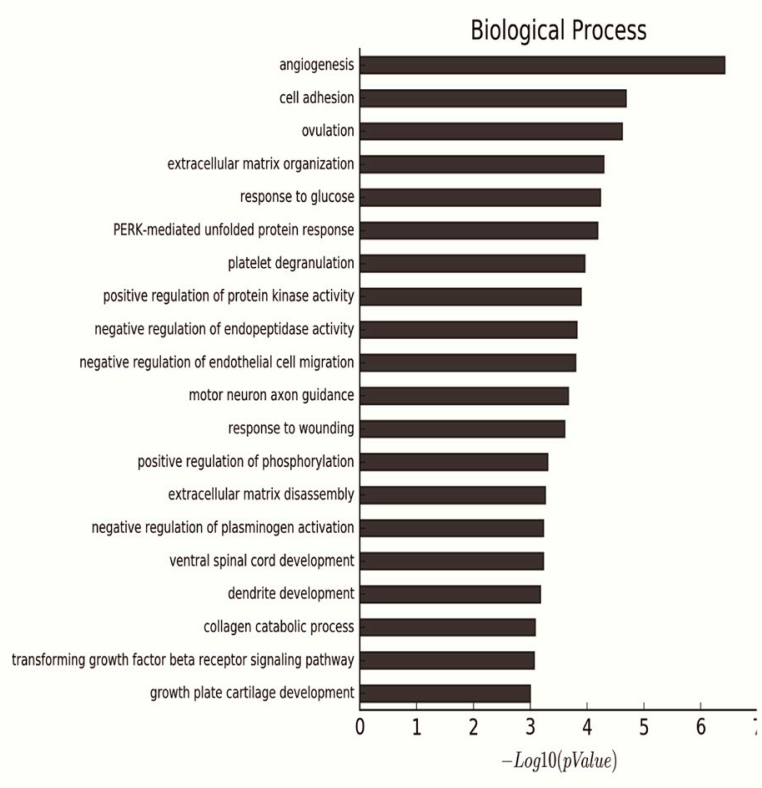

C

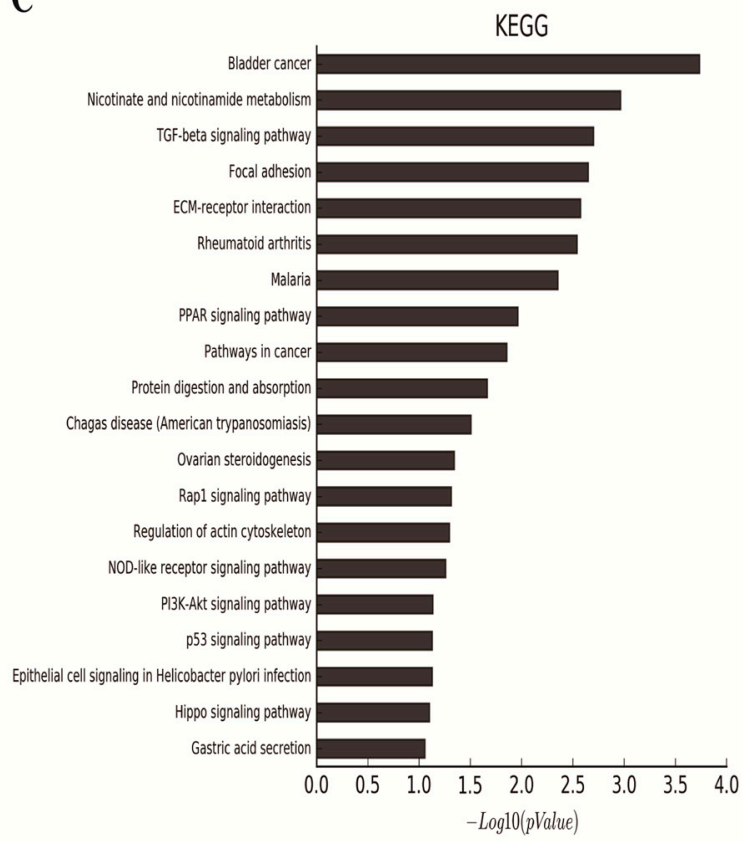

B

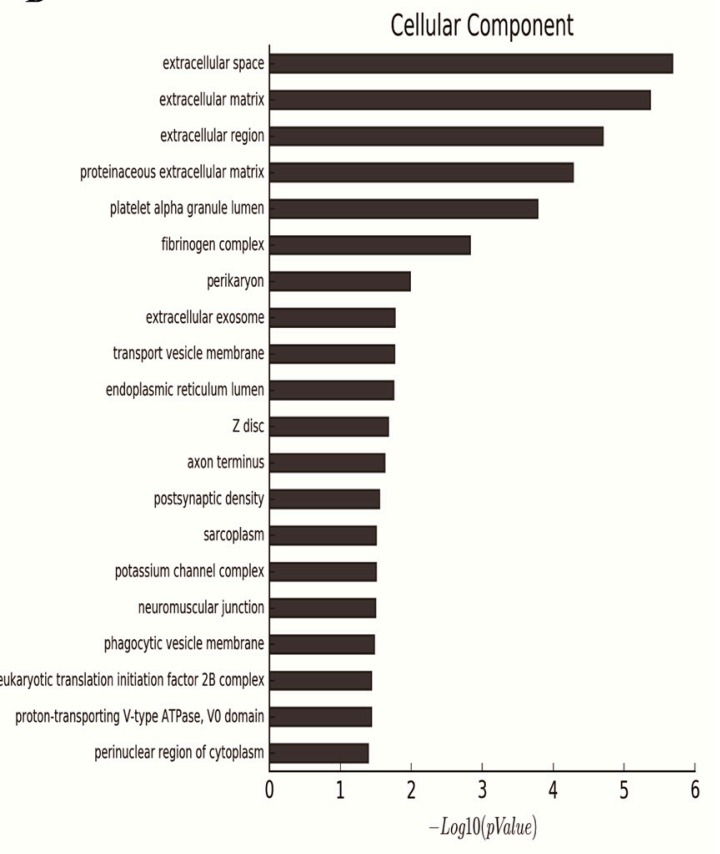

Figure 5. Genome-wide analysis of RHPN1-AS1 targets. (A,B) Significant GOs affected by RHPN1-AS1 silencing. $X$-axis, negative logarithm of the $p$ value $(-\operatorname{Lg} p)$; $Y$-axis, the Gene Ontology (GO) category; (C) Histogram of signal pathways that were regulated by RHPN1-AS1 silencing. $X$-axis, negative logarithm of the $p$-value $(-\operatorname{Lg} p) ; Y$-axis, the name of the pathway.

\section{Discussion}

Melanoma is an aggressive malignant tumor of the melanocytes. Somatic mutations in the RAS/RAF/MEK/ERK signaling pathway are frequent in cutaneous melanomas, with $50 \%-70 \%$ of them being BRAF mutations [16]. However, BRAF mutations are rare in UM, and GNAQ and GNA11 are typically mutated in UM. Thus, it is evident that aberrant signaling pathways in UM may be distinctive. Microarray assays and SNP assays have also confirmed the mutation frequencies of $B A P 1, S F 3 B 1$ and EIF1AX in UM [17]. GNAQ is inversely associated with chromosome 3 monosomy 
and metastasis; and BAP1 is significantly associated with chromosome 3 monosomy but not with relapse [17].

Global transcriptional analyses have revealed that the human genome is transcribed to produce a wide range of lncRNAs [18]. Recently, studies have highlighted that lncRNAs are connected with fundamental characteristics in a variety of tumors [11]. For example, prostate cancer associated transcript 1 (PCAT-1) is highly upregulated in metastatic prostate cancers. PCAT-1 knockdown increases cell proliferation, suggesting that PCAT-1 might contribute to prostate cancer progression [19]. Through genome screening, $\mathrm{Li}$ et al. found that five ncRNA fragments interacted with the tumor suppressor PSF and released it from the proto-oncogene G antigen 6 (GAGE6) regulatory locus, resulting in an activation of GAGE6 expression. Overexpression of these ncRNA fragments in melanoma cell lines led to enhanced tumorigenesis [20]. However, the role for IncRNAs in UM remains obscure. We compared the different expression profiles of lncRNAs between UM samples and normal tissues and identified a large number of aberrantly expressed lncRNAs in UM tissues (data not shown). RHPN1-AS1 was highly expressed in UM tissues and cell lines, and was located at the 8q24.3, a region that is frequently amplified in a wide range of solid tumors, including UM. In this study, we found that the downregulation of RHPN1-AS1 could significantly inhibit proliferation, migration and invasion of UM cells in vitro. Furthermore, the downregulation of RHPN1-AS1 could inhibit tumor growth in murine model. Taken together, these findings indicated that RHPN1-AS1 might serve as an oncoRNA and play an important role in UM progression.

It has been suggested that antisense RNAs could potentially regulate their corresponding sense mRNA at different level [21,22]. RHPN1-AS1 is a $2.03 \mathrm{~kb}$ RNA transcribed from the reverse strand of chromosome 8, on the opposite strand of the protein coding gene RHPN1 (8q24.3), but there is no overlapping and complementary region between RHPN1-AS1 and RHPN1. To determine the relationship between RHPN1-AS1 and RHPN1, we examined the mRNA and protein level of RHPN1 after knocking down endogenous RHPN1-AS1 expression in OCM1 and OM431 cells using qPCR and western blot. The results indicated that down-regulated expression of RHPN1-AS1 did not significantly affect RHPN1 expression (Figure S2), suggesting that the biological effect of RHPN1-AS1 is independent of RHPN1.

Previous studies have shown that not only can lncRNAs govern expression of neighboring protein-coding genes (cis-acting regulation), but also they can regulate distal transcriptional elements (trans-acting) through various mechanisms [23]. Given that expression of RHPN1 did not change after RHPN1-AS1 knockdown, we further explored the genes and pathways that RHPN1-AS1 may regulate using microarray analysis. GO analysis revealed that differentially expressed genes after RHPN1-AS1 knockdown are associated with biological processes including angiogenesis, cell adhesion and extracellular matrix organization. Pathway analysis using the KEGG database revealed that the most significantly changed pathways included bladder cancer, nicotinate and nicotinamide metabolism and TGF- $\beta$ signaling pathway. It is noteworthy that TGF- $\beta$ signaling pathway, which has a tremendous impact on the regulation of EMT is significantly changed after RHPN1-AS1 knockdown. Evidence suggests that epithelial-to-mesenchymal transition (EMT) participates during the progression of cancer, and TGF- $\beta$ signaling promotes EMT by initiating the process and establishing a dramatic cellular adaptation that permeates a great number of vital cell biological processes [24]. Therefore, we hypothesize that RHPN1-AS1 may promote UM progression at least partly through interplay with TGF- $\beta$ signaling pathway. However, the specific cellular processes that are directly affected by alterations in RHPN1-AS1 expression remain to be elucidated.

Accumulating studies have shown that IncRNAs act as crucial regulators in tumor progression $[25,26]$. Therefore, lncRNAs could be one of the leading forces during UM tumorigenesis. Identification of UM-associated lncRNAs and investigation of their biological functions and clinical significance may provide a strategy and facilitate the development of lncRNA-directed diagnosis and improved prognosis of UM. 


\section{Materials and Methods}

\subsection{Cell Culture and Short Interfering RNAs (siRNAs) in UM Cells}

OM431, OCM1 and RPE cells were cultured in Dulbecco's Modified Essential Medium (DMEM; Gibco, Carlsbad, CA, USA) with $10 \%$ fetal bovine serum (FBS) and maintained at $37{ }^{\circ} \mathrm{C}$ in a $5 \%$ $\mathrm{CO}_{2}$ atmosphere. Three different siRNAs targeting RHPN1-AS1 and a scrambled siRNA mock were designed and synthesized (Biotend, Shanghai, China). In total, $2 \times 10^{5} \mathrm{UM}$ cells were seeded into six-well plates and transfected with the siRNAs using Lipofectamine 2000 (Invitrogen, Carlsbad, CA, USA) according to the manufacturer's protocol. The nucleotide sequences of the siRNAs for RHPN1-AS1 are shown in Table 1. Transfection efficiency was optimized using fluorescein labeled negative controls. Cells were harvested at $48 \mathrm{~h}$.

Table 1. Primers and siRNA used in this study.

\begin{tabular}{|c|c|c|}
\hline Primer Name & Sequence $\left(5^{\prime}-3^{\prime}\right)$ & Purpose \\
\hline RHPN1-AS1-F & GCTCCTGGTCATCAAGTTCCTCT & qRT-PCR \\
\hline RHPN1-AS1-R & GCACAGGCACCAGAATGATCC & qRT-PCR \\
\hline RHPN1-F & TACGACTCGCTTACTGGGGT & qRT-PCR \\
\hline RHPN1-R & GAGGGCACCGATGTTGAAGA & qRT-PCR \\
\hline GAPDH-F & AGGTCGGAGTCAACGGATTTG & qRT-PCR \\
\hline GAPDH-R & TGTAAACCATGTAGTTGAGGTCA & qRT-PCR \\
\hline RHPN1-AS1-si1-sense & CCGAAUCUCUUUACUUCCAdTdT & siRHPN1-AS1 \\
\hline RHPN1-AS1-si1-antisense & UGGAAGUAAAGAGAUUCGGdTdT & siRHPN1-AS1 \\
\hline RHPN1-AS1-si2-sense & CUCAAACUUUGAGGGUCAUdTdT & siRHPN1-AS1 \\
\hline RHPN1-AS1-si2-antisense & AUGACCCUCAAAGUUUGAGdTdT & siRHPN1-AS1 \\
\hline RHPN1-AS1-si3-sense & CUUCCAUACUUCCCUAGGUdTdT & siRHPN1-AS1 \\
\hline RHPN1-AS1-si3-antisense & ACCUAGGGAAGUAUGGAAGdTdT & siRHPN1-AS1 \\
\hline
\end{tabular}

\subsection{RNA Extraction and Quantitative Real-Time Polymerase Chain Reaction (qRT-PCR) Assays}

Total RNA was isolated using Trizol reagent (Invitrogen) following the manufacturer's protocol. For qRT-PCR, RNA was reverse transcribed to cDNA using a Reverse Transcription Kit (Takara, Dalian, China). Screening for lncRNAs was performed by qRT-PCR with the primer sets described in Table 1. Real-time PCR analyses were performed using SYBR Premix Ex Taq (Takara), which was repeated at least three times for each sample. Gene expression levels were normalized to that of GAPDH. The qRT-PCR assays were conducted on a Roche PCR instrument.

\subsection{FluorescenceIn Situ Hybridization}

RHPN1-AS1-Fish Probe Mix was purchased from RiboBio Corporation (Guangzhou, China), and Fluorescence in situ hybridization was performed using Fluorescent In Situ Hybridization Kit (RiboBio Co., Guangzhou, China). Briefly, OCM1 cells grown on cover slips were fixed with 4\% formaldehyde for $10 \mathrm{~min}$, and then permeabilized with $0.5 \%$ Triton X-100 in phosphate-buffered saline (PBS) for $20 \mathrm{~min}$. For hybridization, the cells were incubated in hybridization mixtures at $37^{\circ} \mathrm{C}$ for $30 \mathrm{~min}$ before probes were added. After incubation with RHPN1-AS1-Fish Probe Mix at $37^{\circ} \mathrm{C}$ overnight, the cells were washed with $4 \times$ saline sodium citrate (SSC; containing $0.1 \%$ Tween-20) at $42{ }^{\circ} \mathrm{C}$ for $5 \mathrm{~min}$, followed by $2 \times \mathrm{SSC}$ at $42{ }^{\circ} \mathrm{C}$ for $5 \mathrm{~min}$ and $1 \times \mathrm{SSC}$ at $42{ }^{\circ} \mathrm{C}$ for $5 \mathrm{~min}$. The nuclei were counterstained with $4^{\prime}$,6-diamidino-2-phenylindole (DAPI), after which images were taken.

\subsection{Short Hairpin (sh) RNA-Expressing Plasmid Construction, Lentivirus Packaging, Cloning and} Stable Transfection

To reduce the expression of RHPN1-AS1, two human RHPN1-AS1 shRNA sequences $5^{\prime}$-CCGAATC TCTTTACTTCCA-3' and 5'-CTCAAACTTTGAGGGTCAT-3' were cloned into the pGIPZ-lentivirus vector (System Biosciences, Palo Alto, CA, USA). Thereafter, two RHPN1-AS1 knockdown vectors, 
namely pGIPZ-RHPN1-AS1-sh1 and pGIPZ-RHPN1-AS1-sh2, were constructed and sequenced (Figure S3). The empty pGIPZ vector without any insertion was used as a control. 293T cells were cultured in DMEM containing $10 \% \mathrm{FBS}$, maintained at $37^{\circ} \mathrm{C}$ and transfected using Lipofectamine 2000 reagent (Invitrogen, Carlsbad, CA, USA) with $3 \mu \mathrm{g}$ pGIPZ-RHPN1-AS1-sh1 or -sh2, $6.0 \mu \mathrm{g}$ PsPax2 and $3 \mu \mathrm{g}$ pMD 2.G. The media were replaced with $10 \mathrm{~mL}$ fresh medium after incubation overnight. The virus-containing supernatants were collected at 48 and $72 \mathrm{~h}$. OCM1 and OM431 cells were infected and then selected using $4 \mu \mathrm{g} / \mathrm{mL}$ puromycin. The knockdown efficiency was measured by qRT-PCR.

\subsection{Cell Migration Assay}

Totally, $1 \times 10^{5}$ cells in serum-free media were placed into the upper chamber of each insert $(8.0 \mu \mathrm{m}$, Millipore, Palo Alto, MA, USA). Then the chambers were incubated for $36 \mathrm{~h}$ in $600 \mu \mathrm{L}$ culture medium supplemented with $10 \%$ FBS in the bottom chambers before examination. The cells on the upper surface were scraped off with a cotton swab, and the migrated cells on the lower surface were fixed $4 \%$ paraformaldehyde and stained with $5 \%$ crystal violet. After washing with PBS, images were taken. Experiments were independently repeated in triplicate.

\subsection{Cell Invasion Assay}

Matrigel-coated chambers (BD Biosciences, Palo Alto, CA, USA) with $8 \mu \mathrm{m}$ pores were used for invasion assay. Two hundred microliters cells in serum-free medium were seeded in the upper chambers at $5 \times 10^{4} / \mathrm{mL}$ concentration. The lower chamber was filled with $800 \mu \mathrm{L}$ DMEM medium containing $10 \% \mathrm{FBS}$. After incubation at $37^{\circ} \mathrm{C}$ for $24 \mathrm{~h}$, non-invaded cells on the top of the wells were scraped off, whereas the translocated cells on the lower surface were fixed with $4 \%$ paraformaldehyde and stained with $5 \%$ crystal violet. After washing with PBS, cells were counted under a light microscope (Olympus, Tokyo, Japan).

\subsection{Colony Formation Assay}

In total, $2 \times 10^{3}$ cells were placed in each well of the six-well plates and maintained in DMEM containing $10 \% \mathrm{FBS}$ at $37^{\circ} \mathrm{C}$ for 8 days. After 8 days, the cells were fixed with $4 \%$ paraformaldehyde and stained with $5 \%$ crystal violet. Visible colonies were then counted and imaged. Experiments were independently repeated three times.

\subsection{Tumor Xenograft Model in Nude Mice}

OCM1 cells stably expressing RHPN1-AS1-sh1 and control OCM1 cells $\left(1 \times 10^{6}\right)$ were subcutaneously injected at $100 \mu \mathrm{L}$ cell suspension per injection into the right flanks of 3-week-old athymic nude mice ( $n=6$ per group). The mice were housed under a controlled environment in a sterile facility. The tumor volume was measured once every 3-4 days with calipers. Tumor volume was calculated using the following formula: $0.5 \times$ length $\times$ width $\times$ height. After 28 days, the mice were sacrificed, and the tumors were removed and analyzed. The Animal Care and Use Committee at Ninth People' Hospital affiliated to Shanghai Jiao Tong University, School of Medicine approved the animal protocols (HKDL[2014]70, 25 February 2014).

\subsection{Nuclear/Cytoplasmic RNA Fractionation}

OCM1 cells were grown in $10 \mathrm{~cm}$ dishes. After reaching $80 \%$ confluence, cells were centrifuged at $800 \mathrm{rpm}$ for $5 \mathrm{~min}$ and rinsed with ice-cold PBS. Nuclear/cytoplasmic RNA fractionation was performed using Nuclear/Cytosol Fractionation Kit (BioVision Inc., Milpitas, CA, USA) according to manufacturer's protocol. After cytoplasmic fraction and nuclear fraction were isolated, RNAs were extracted by Trizol reagent (Invitrogen). Semi-quantitative RT-PCR was then performed to evaluate the relative abundance of RHPN1-AS1, U1 and GAPDH in each sample. 


\subsection{DNA Microarray Analysis and Data Analysis}

The AffymetrixPrimeView ${ }^{\mathrm{TM}}$ Human Gene Expression Array (Affymetrix, Santa Clara, CA, USA) was used in this experiment. Total RNA of OCM1 cells and OCM1 cells stably transfected with RHPN1-AS1-sh1 was isolated using the TRIzol reagent (Invitrogen, Carlsbad, CA, USA). Total RNA was quantified using NanoDrop ND-2000 (Thermo Scientific, Waltham, MA, USA) and the RNA integrity was assessed by the Agilent Bioanalyzer 2100 (Agilent Technologies, Santa Clara, CA, USA). The sample labeling, microarray hybridization and washing were performed according to the manufacturer's standard protocols. Briefly, total RNA were transcribed to double strand cDNA, then synthesized cRNA and labeled with biotin. The labeled cRNAs were hybridized onto the microarray. After washing and staining, the arrays were scanned by the Affymetrix Scanner 3000 (Affymetrix, Santa Clara, CA, USA). Affymetrix GeneChip Command Console (version 4.0, Affymetrix) was employed to analyze array images to get raw data, which was then analyzed using Genesping software (version 13.1; Agilent Technologies). First, the raw data was normalized with the RMA algorithm. Through fold change and $\mathrm{p}$ value calculated by $t$-test, differentially expressed genes were identified. A threshold of fold change $\geq 2.0$ and $p$-value $\leq 0.05$ was set for identifying up- and down-regulated genes. Afterwards, Gene Ontology (GO) database and Kyoto Encyclopedia of Genes and Genomes (KEGG) database were used for the functional annotation and enrichment analysis of these differentially expressed mRNAs.

\subsection{Statistical Analysis}

All statistical analyses were performed using SPSS version 19.0 software (SPSS, Inc., Chicago, IL, USA). $p$-values $<0.05$ were considered to be statistically significant.

\section{Conclusions}

In this study, we verified that RHPN1-AS1 was upregulated in UM cancerous tissues and plays a vital role in UM cell proliferation, migration and invasion. Depletion of RHPN1-AS1 inhibited the growth of UM in vitro and in vivo. Microarray analysis further identified multiple pathways and biological processes that might be responsible for UM tumor growth and metastasis that were altered by RHPN1-AS1. These results suggest RHPN1-AS1 may be an attractive biomarker and therapeutic target in UM.

Supplementary Materials: Supplementary materials can be found at www.mdpi.com/1422-0067/18/1/226/s1.

Acknowledgments: This work was supported by the National Natural Science Foundation of China (Grants 81500757, 81502481, 81372909, 81570884, 31470757, 81372469, U1432117 and 81602366 ), the Scientific Research Program of National Health and Family Planning Commission of China (2014040), the Program for Professor of Special Appointment (Eastern Scholar) at the Shanghai Institutions of Higher Learning (1410000159), the "Dawn" Program of Shanghai Education Commission (14SG18), the SMC-ChenXing Yong Scholar Program (2014, Class B), the Shanghai Municipal Education Commission-Gaofeng Clinical Medicine Grant (20161317), the Science and Technology Commission of Shanghai (Grants 14JC1404100, 14JC1404200, and 14430723100), the Outstanding Yong Scholar Grant of Shanghai Jiao Tong University School of Medicine (16XJ11002), the Foundation of Ninth People's Hospital Affiliated to Shanghai Jiao Tong University School of Medicine (2014A05) and the Shanghai Jiao Tong University School of Medicine Doctor Innovation Fund (BXJ201432).

Author Contributions: Linna Lu, He Zhang, Renbing Jia, Shengfang Ge and Xianqun Fan conceived and designed the experiments. Xiaoyu Yu, Shiqiong Xu, Yue Xing and Jiayan Fan were involved in the provision of study material. Linna Lu, Xuyang Wen, Xia Ding, Hui Pan and Leilei Zhang analyzed and interpreted the data. Linna Lu, Xiaoyu Yu and Leilei Zhang wrote the manuscript. Linna Lu, Renbing Jia, He Zhang and Xianqun Fan approved the final version. All authors read and approved the final manuscript.

Conflicts of Interest: The authors declare no conflict of interest.

\section{References}

1. Bol, K.F.; Mensink, H.W.; Aarntzen, E.H.; Schreibelt, G.; Keunen, J.E.; Coulie, P.G.; de Klein, A.; Punt, C.J.; Paridaens, D.; Figdor, C.G.; et al. Long Overall Survival after Dendritic Cell Vaccination in Metastatic Uveal Melanoma Patients. Am. J. Ophthalmol. 2014, 158, 939-947. [CrossRef] [PubMed] 
2. Rietschel, P.; Panageas, K.S.; Hanlon, C.; Patel, A.; Abramson, D.H.; Chapman, P.B. Variates of Survival in Metastatic Uveal Melanoma. J. Clin. Oncol. 2005, 23, 8076-8080. [CrossRef] [PubMed]

3. Diener-West, M.; Reynolds, S.M.; Agugliaro, D.J.; Caldwell, R.; Cumming, K.; Earle, J.D.; Hawkins, B.S.; Hayman, J.A.; Jaiyesimi, I.; Jampol, L.M.; et al. Development of Metastatic Disease after Enrollment in the Coms Trials for Treatment of Choroidal Melanoma: Collaborative Ocular Melanoma Study Group Report No. 26. Arch. Ophthalmol. 2005, 123, 1639-1643. [PubMed]

4. Buzzacco, D.M.; Abdel-Rahman, M.H.; Park, S.; Davidorf, F.; Olencki, T.; Cebulla, C.M. Long-Term Survivors with Metastatic Uveal Melanoma. Open Ophthalmol. J. 2012, 6, 49-53. [CrossRef] [PubMed]

5. Furney, S.J.; Pedersen, M.; Gentien, D.; Dumont, A.G.; Rapinat, A.; Desjardins, L.; Turajlic, S.; Piperno-Neumann, S.; de la Grange, P.; Roman-Roman, S.; et al. SF3B1 Mutations Are Associated with Alternative Splicing in Uveal Melanoma. Cancer Discov. 2013, 3, 1122-1129. [CrossRef] [PubMed]

6. Van Raamsdonk, C.D.; Griewank, K.G.; Crosby, M.B.; Garrido, M.C.; Vemula, S.; Wiesner, T.; Obenauf, A.C.; Wackernagel, W.; Green, G.; Bouvier, N.; et al. Mutations in GNA11 in Uveal Melanoma. N. Engl. J. Med. 2010, 363, 2191-2199. [CrossRef] [PubMed]

7. Harbour, J.W.; Onken, M.D.; Roberson, E.D.; Duan, S.; Cao, L.; Worley, L.A.; Council, M.L.; Matatall, K.A.; Helms, C.; Bowcock, A.M. Frequent Mutation of BAP1 in Metastasizing Uveal Melanomas. Science 2010, 330, 1410-1413. [CrossRef] [PubMed]

8. Hauptman, N.; Glavac, D. Long Non-Coding RNA in Cancer. Int. J. Mol. Sci. 2013, 14, 4655-4669. [CrossRef] [PubMed]

9. Djebali, S.; Davis, C.A.; Merkel, A.; Dobin, A.; Lassmann, T.; Mortazavi, A.; Tanzer, A.; Lagarde, J.; Lin, W.; Schlesinger, F.; et al. Landscape of Transcription in Human Cells. Nature 2012, 489, 101-108. [CrossRef] [PubMed]

10. Guttman, M.; Amit, I.; Garber, M.; French, C.; Lin, M.F.; Feldser, D.; Huarte, M.; Zuk, O.; Carey, B.W.; Cassady, J.P.; et al. Chromatin Signature Reveals over a Thousand Highly Conserved Large Non-Coding RNAs in Mammals. Nature 2009, 458, 223-227. [CrossRef] [PubMed]

11. Gutschner, T.; Diederichs, S. The Hallmarks of Cancer: A Long Non-Coding RNA Point of View. RNA Biol. 2012, 9, 703-719. [CrossRef] [PubMed]

12. Fan, J.; Xing, Y.; Wen, X.; Jia, R.; Ni, H.; He, J.; Ding, X.; Pan, H.; Qian, G.; Ge, S.; et al. Long Non-Coding RNA Ror Decoys Gene-Specific Histone Methylation to Promote Tumorigenesis. Genome Biol. 2015, 16, 139. [CrossRef] [PubMed]

13. Takayama, K.; Horie-Inoue, K.; Katayama, S.; Suzuki, T.; Tsutsumi, S.; Ikeda, K.; Urano, T.; Fujimura, T.; Takagi, K.; Takahashi, S.; et al. Androgen-Responsive Long Noncoding RNACTBP1-as Promotes Prostate Cancer. EMBO J. 2013, 32, 1665-1680. [CrossRef] [PubMed]

14. Xie, M.; Sun, M.; Zhu, Y.N.; Xia, R.; Liu, Y.W.; Ding, J.; Ma, H.W.; He, X.Z.; Zhang, Z.H.; Liu, Z.J.; et al. Long Noncoding RNA HOXA-AS2 Promotes Gastric Cancer Proliferation by Epigenetically Silencing P21/PLK3/DDIT3 Expression. Oncotarget 2015, 6, 33587-33601. [PubMed]

15. Calin, G.A.; Liu, C.G.; Ferracin, M.; Hyslop, T.; Spizzo, R.; Sevignani, C.; Fabbri, M.; Cimmino, A.; Lee, E.J.; Wojcik, S.E.; et al. Ultraconserved Regions Encoding ncRNAs Are Altered in Human Leukemias and Carcinomas. Cancer Cell 2007, 12, 215-229. [CrossRef] [PubMed]

16. Sarkar, D.; Leung, E.Y.; Baguley, B.C.; Finlay, G.J.; Askarian-Amiri, M.E. Epigenetic Regulation in Human Melanoma: Past and Future. Epigenetics 2015, 10, 103-121. [CrossRef] [PubMed]

17. Dono, M.; Angelini, G.; Cecconi, M.; Amaro, A.; Esposito, A.I.; Mirisola, V.; Maric, I.; Lanza, F.; Nasciuti, F.; Viaggi, S.; et al. Mutation Frequencies of GNAQ, GNA11, BAP1, SF3B1, EIF1AX and TERT in Uveal Melanoma: Detection of an Activating Mutation in the TERT Gene Promoter in a Single Case of Uveal Melanoma. Br. J. Cancer 2014, 110, 1058-1065. [CrossRef] [PubMed]

18. Lee, J.T. Epigenetic Regulation by Long Noncoding Rnas. Science 2012, 338, 1435-1439. [CrossRef] [PubMed]

19. Prensner, J.R.; Iyer, M.K.; Balbin, O.A.; Dhanasekaran, S.M.; Cao, Q.; Brenner, J.C.; Laxman, B.; Asangani, I.A.; Grasso, C.S.; Kominsky, H.D.; et al. Transcriptome Sequencing across a Prostate Cancer Cohort Identifies PCAT-1, an Unannotated LincRNA Implicated in Disease Progression. Nat. Biotechnol. 2011, 29, 742-749. [CrossRef] [PubMed]

20. Li, L.; Feng, T.; Lian, Y.; Zhang, G.; Garen, A.; Song, X. Role of Human Noncoding RNAs in the Control of Tumorigenesis. Proc. Natl. Acad. Sci. USA 2009, 106, 12956-12961. [CrossRef] [PubMed] 
21. Lapidot, M.; Pilpel, Y. Genome-Wide Natural Antisense Transcription: Coupling Its Regulation to Its Different Regulatory Mechanisms. EMBO Rep. 2006, 7, 1216-1222. [CrossRef] [PubMed]

22. Mercer, T.R.; Mattick, J.S. Structure and Function of Long Noncoding RNAs in Epigenetic Regulation. Nat. Struct. Mol. Biol. 2013, 20, 300-307. [CrossRef] [PubMed]

23. Moran, V.A.; Perera, R.J.; Khalil, A.M. Emerging Functional and Mechanistic Paradigms of Mammalian Long Non-Coding RNAs. Nucleic Acids Res. 2012, 40, 6391-6400. [CrossRef] [PubMed]

24. Moustakas, A.; Heldin, C.H. Mechanisms of Tgf $\beta$-Induced Epithelial-Mesenchymal Transition. J. Clin. Med. 2016, 5, 63. [CrossRef] [PubMed]

25. Walsh, A.L.; Tuzova, A.V.; Bolton, E.M.; Lynch, T.H.; Perry, A.S. Long Noncoding RNAs and Prostate Carcinogenesis: The Missing "Linc"? Trends Mol. Med. 2014, 20, 428-436. [CrossRef] [PubMed]

26. Kotake, Y.; Nakagawa, T.; Kitagawa, K.; Suzuki, S.; Liu, N.; Kitagawa, M.; Xiong, Y. Long Non-Coding RNA ANRIL Is Required for the PRC2 Recruitment to and Silencing of P15(INK4b) Tumor Suppressor Gene. Oncogene 2011, 30, 1956-1962. [CrossRef] [PubMed]

(C) 2017 by the authors; licensee MDPI, Basel, Switzerland. This article is an open access article distributed under the terms and conditions of the Creative Commons Attribution (CC BY) license (http:/ / creativecommons.org/licenses/by/4.0/). 